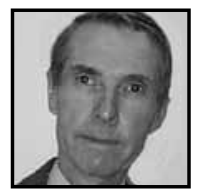

\title{
Brain, Mind, and the Organization of Knowledge for Effective Recall and Application
}

\author{
O. Roger Anderson, Columbia University
}

\begin{abstract}
Modern neuroscientific research has confirmed and amplified our understanding of how we learn and subsequently recall information from memory. This paper presents a novel model of information networking in memory based on neurocognitive theory. Implications for teaching and learning are discussed-especially ways of enhancing learning of subject matter that promote its effective recall, logical organization in networks, and effective application to thinking, including problem solving and higher-order cognitive processes.
\end{abstract}

\section{Introduction}



challenge in curriculum design and teaching is to enhance students' ability to organize information in a way that makes it available for efficient recall in response to an appropriate context, and with sufficient generality to be applied in new situations (e.g., critical thinking and problem solving). Increasing emphasis in modern society is placed on enhancing public literacy of major ideas in the disciplines, especially an ability to explain our thoughts and to mobilize arguments to support well-reasoned positions. This is particularly true in the sciences, where knowledge of fundamental scientific principles may be critical in democratic decision-making about technology, management of the environment, and the role of science in society. However, it is also an ideal relevant to the liberal arts disciplines. A liberally educated person should be able to engage in conversation about some major ideas pertinent to cultural, historical, and social domains in a way that is informed and contextually relevant. 
We all have probably had the perplexing experience of teaching a major topic in a way that seemed to be immediately effective, only to discover later that some of the students were able to recall and mobilize the information in a thorough and relevant way relative to the context we provided (e.g., a discussion topic, test item, etcetera), while other students seemed unable to recall much of anything, or only fragmented aspects. Why do some learning experiences yield relatively lasting effective organization of knowledge in memory, while others at least for some students appear to yield partial or fleeting recall? There is no solitary or simple explanation for these individual differences in knowledge recall and its application, nor is there a foolproof prescription for teaching that will improve performance. However, a recent line of research is explored in this paper that addresses these issues within a perspective of some current knowledge of the interrelationships of brain, mind, and organization of information in memory, especially focusing on the role of knowledge networks in memory and their dynamic mobilization and application within particular contexts.

A knowledge network in memory is defined generally as the organization of meaningful information within a multi-relational logical framework in a way that permits effective internal reflection and public communication. More specifically, the purpose of this paper is to explore some modern evidence on how we organize and encode information in memory, and how the brain functions to mediate recall and organization of information in response to contextual cues that occasion relevant knowledge recall and its application. Neuroscientific research on how we think and reason is growing exponentially. Only sufficient information about brain structure and function is presented as pertinent to the focus of this paper, and largely in a descriptive format. Practical applications to teaching and learning are emphasized.

\section{Memory, Remembering, and Knowing}

As background, it is important to distinguish at the outset between three cognitive functions: (1) Memory, (2) Remembering, and (3) Knowing. Memory is a state of mind-a store of potentially retrievable information that has been encoded in a sufficiently stable way to be accessed at a later time. Remembering is a mental process of actively mobilizing and reconstructing stored information in a way that makes it accessible for recall relative to a set of contextual cues. Knowing is a conscious disposition of organizing information in such a way that it represents a coherent exposition-one that is meaningful to the person who assembles it and capable of being 
shared with others. For example, in contrast to meaningful remembering, experimental psychologists have utilized nonsense syllables (strings of a few alphabet letters that have little or no possible resemblance to words) as a way of studying memory. The nonsense syllables are intended to be memorized and recalled as a way of examining "pure memory functions," without the contamination of prior knowledge. We could certainly say that the nonsense syllables were stored in memory and that they can be remembered. It would not be appropriate to state that we "know them." They are not meaningful constructs of knowledge.

\section{The Roles of Working Memory and Long-Term Memory}

We also need to clarify the difference between "short-term memory" (also known as "working memory" in modern usage) and "long-term memory." Working memory mobilizes information from long-term memory to make it accessible to be "worked with."That is, to actively organize information, interrelate it, or otherwise prepare it for a purpose: e.g., application to a task, problem solving, communication, or by internal reflection to enlarge our long-term memory (e.g., Gathercole \& Alloway, 2008; Thorne, 2006). Long-term memory is the information we have committed to long-term storage for retrieval at a later time. Working memory is dynamic and typically of short duration (seconds to minutes). Long-term memory is more stable and can persist for a lifetime. For example, suppose someone asks us to recommend a student who would be a good candidate for an academically gifted learning experience. Assuming that we have stored some fundamental information about most of our students, we probably would begin to mobilize and review in working memory as many visual and semantic (verbal) sources of information about students that are accessible from long-term memory. Implicitly, or more explicitly, we may begin to apply some criteria for assessing the academically gifted and begin to assemble a list of students who we conclude meet the criteria. The process of accessing long-term memory, mobilizing information, and reflectively processing it to reach a goal is accomplished in working memory-a dynamic state of mind.

Sufficient modern scientific evidence has been gathered to explain basically where and how these two forms of memory occur in our brains (see Figure 1 below). 




Fig. 1: Human cerebral cortex ${ }^{1}$

We will avoid details of some current theoretical controversies (e.g., Jonides et al., 2008), and focus on consensus interpretations. There is good evidence from neuro-imaging (e.g., MRI) and other brain monitoring techniques that much of the dynamic process of organizing and reflectively thinking about information in working memory is mediated by the frontal lobes (immediately behind our forehead). The frontal lobes, however, are reciprocally connected (forward and backward) by nerve fibers to other parts of the brain (e.g., Figure 1). These include the back of the brain (occipital lobe) where visual images are perceived and stored in memory, the temporal lobes (immediately inside both sides of the skull, opposite the temples behind the eyes) where verbal and conceptual knowledge is partially stored, and the somatosensory cortex (at the top of the brain posterior to the frontal lobes) where sensations of touch and body sensations are located. There are other areas of the cortex, also interconnected with the frontal lobes, where auditory percepts are stored (auditory cortex). The nerve fiber interconnections permit the frontal lobes to access information stored as memories within each of these brain centers. The frontal lobes also exert organizational control over the access and processing of stored information. One of the significant control (executive) functions of the frontal lobes is focal attention. That is, the frontal lobes have been shown to be active when we localize information in memory for specific attention. For example, if we were asked to recall a visual image of the map of North America, we likely would mobilize a general mental image of the overall geography of North America, especially major features of continental boundaries surrounding Canada and the United States, but initially with less focus on details. With intent, however, we can specifically recall details, such as focusing in on the Great Lakes - the details varying depending on our familiarity with their geography.

This capacity to voluntarily focus on specific details, versus general recall of visual information, is mediated by frontal lobe activity through nerve tracts projecting from the frontal lobes back toward the visual cortex. During recall of visual 
information in the occipital lobe, visual memories are reactivated in the same regions where they were initially perceived. The particular images recalled and their focal detail is controlled by impulses from the frontal lobes transmitted through the nerve fibers projecting back to the occipital lobe. There are other major nerve tracts interconnecting many parts of the brain. These rich arrays of interlinking nerve tracts, among other functions, account for the holistic way that the brain coordinates information processing. In addition to these important linkages, the frontal lobes are also interconnected with a deeper region of the brain known as the limbic system. It has multiple roles. One is to help us consolidate new information to be stored in longterm memory. Another area of the limbic system mediates our emotional or affective responses. Hence, the frontal lobe can access and partially moderate our emotional sensations during information processing. We will refer to this as affective functions. We can now assemble an educationally relevant theoretical model of how we dynamically organize and interrelate information in memory to enhance the networking of information during recall and its application.

\section{A "Contextual-Label-Pointer" (CLP) Model of Information Networking in Memory}

Human brain functions are complex, diversified and highly adaptable, especially with respect to information processing and cognitive representations of experience (e.g., Anderson, 2009b; Holistic-educator, n.d.). The following theoretical discussion is not intended to be a general theory of cognition, rather it is a theoretical model that may have practical value in education, especially as a novel way of understanding how learners organize and recall networks of information in memory.

Returning to our previously cited example of recalling a visual image of the map of North America, assume that we were asked to describe our knowledge of the Great Lakes. If we are a resident of Quebec, Canada, we may well identify Lake Ontario as a starting point and begin a narrative by relating it to the other lakes. However, if we are a resident of Chicago, Lake Michigan may well be the starting point. Not necessarily so, of course, if the context for the request specifically states, "Describe the Great Lakes beginning with the largest one." Lake Superior as the name implies is the largest, and there is a definite order of size relationships: i.e., Superior $\rightarrow$ Huron $\rightarrow$ Michigan $\rightarrow$ Erie $\rightarrow$ Ontario. According to current neurocognitive theory, we mobilize this information in long-term memory by frontal lobe activity relative to the contextual cues that are provided to us. Within a given context, the frontal lobes also mobilize organizing rules or principles to guide the construction of information in working memory. In this case, the rule is size relationships. If we were asked to make 
a reply based on a different rule, such as which lakes are most closely related to the geographic boundaries of Canada, we would likely create a different answer. Again, context is a major factor in determining how the frontal lobes activate major organizing rules or principles to guide the assembly of information in working memory. In a coherent assembly of information, our response may contain (among other aspects) characteristics of, and relationships among, the recalled entities.

The way we construct relationships among recalled items from memory to be communicated as narrative is particularly the focus of this paper. The sequential arrangement of these multiple relationships is one of the cardinal proxies (evidence) for networking of knowledge in memory. How can we create a model to represent the organization and processing of information during a coherent, interrelated, exposition of recalled information? Fundamentally, the concepts of information labels and pointers are introduced and more fully explicated below to explain information networking. A hypothetical example is diagrammed in Figure 2.

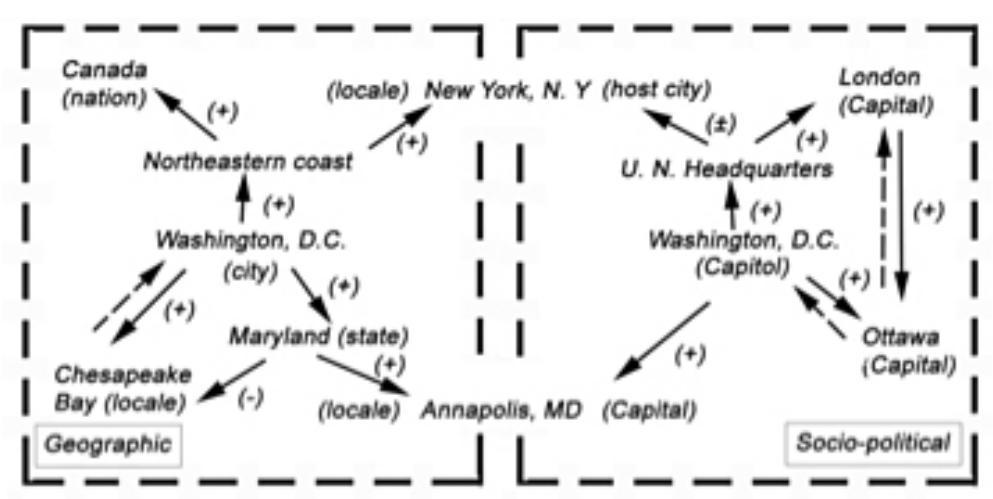

Fig. 2: Interconnected information in networks ${ }^{2}$

When an item of meaningful information is recalled in working memory it may be represented by a "label." A label is a word and/or other symbolic representation (e.g., an iconic visual tag) that is used to represent an entity or category of information in working memory to be assembled as a network. In Figure 2, the verbal label "Washington, D.C." is a placeholder for information that we may have available in memory about the Capitol of the U.S.A. It can represent a specific unit of information, e.g., a declarative sentence "Washington, D.C. is the Capitol of the U.S.A." Or it may represent a category of information, including all of the relevant fundamental information about the city, its geographic locale, culture, history, role in U.S. government, etcetera. In the model, we presume that such labels in working memory also 
are associated with organizational "pointers." A pointer is a mediating adjunct to a label, previously learned. It is used in working memory to point toward, or to indicate, what other entities of information can be accessed in making logical linkages. The larger the number of pointers available in memory for a given labeled entity, the greater the likelihood that the person can construct a more elaborate and coherently organized network of information. However, the particular pointers mobilized will likely depend on the context. That is, from among the total repertoire of accessible pointers only those that are most relevant to the pertinent contextual cues are likely to be mobilized and used. In Figure 2, there are two examples of how the label of "Washington, D.C." may be recalled within two different contexts: socio-political (right panel) and geographic (left panel). Each arrow represents a pointer that potentially can be used to make a relationship of the idea of "Washington, D.C." with other ideational units. Note that each pointer also has either a $(+),(-)$ or $( \pm)$. Each indicates the dominant emotional valence or emotional feeling associated with the linkage. $A(+)$ indicates a positive emotional association and (-) a negative connotation. $A( \pm)$ is used to indicate neutral affect. Moreover, the pointer indicates the most likely direction of association in assembling a narrative sequence. Pointers, however, may be bi-directional (e.g., dashed arrows, Figure 2), indicating that the linkages may likely go either way depending on the logical framework and flow of the narrative being constructed in working memory. The larger the number of bi-directional arrows in the person's repertoire of potential linkages, the more likely the person will construct a richer and more interwoven (inter-linked) set of ideas in her/his assembled network of knowledge.

Working memory is limited in capacity, and labels that represent categories of information are more likely to provide efficiency in organizing and interrelating information in working memory compared to labels that represent only limited information. During the assembly process, the pointers are the potential linkages that can be selected to create a networked set of ideas. In the case of the socio-political context, other labeled entities that are connected by pointers from "Washington, D.C." represent particular subsets of information that can be recalled and in a particular sequence. Note that the other labels in the socio-political context have modifiers (in parentheses) to indicate the contextual category that is relevant to the socio-political perspective, such as (national capitol), etcetera. By comparison, the entities in the geographic contextual field have modifiers that are relevant to geographic concepts (locale, city, etcetera). Two labeled entities (bearing appropriate modifiers for each context) are convergent, that is, accessible within both contexts. They are the labels for the cities of New York and Annapolis (spanning both contextual fields). Note, however, the modifier in parentheses for each one is different depending on the context. 
Examining the network of labels and pointers, it is possible to imagine how the organization of the information would unfold when communicated by the person. For example, let's examine the socio-political context. Beginning with the idea of Washington, D.C., using the pointers may lead to the national role of the U.S. in the U.N., located in the host city of New York (NYC), including representatives from London (U.K. capital) and others in the Commonwealth including Ottawa, where officials are in communication with London, etcetera. The reciprocal dashed arrow indicates a possible recall that Ottawa in North America would likely be in communication with Washington, D.C., located in Maryland, and that the U.S. government also must have some political linkages to the state capital (Annapolis). The location of the labels for NYC and Annapolis, at the boundaries between the two contexts, indicates that these ideational units can act as bridges between the two contexts. This example of coherent and cross-connected discourse contrasts with communications where there is less evidence of inter-linked narrative. For example, some students orally, or in writing, seem to recall information largely as a stream of unconnected declarative sentences. Such sequential, non-networked, narrative is often marked by discontinuous logical connectives and little evidence of a coherent overall perspective. Such narrative is categorized here as having low network organization. We will not comment on the affective valence markers for now, but merely point out that the (-) marker on the link to the Chesapeake Bay may indicate, for example, a concern about pollution of the Bay. The ambiguous marker ( \pm ) for the link from U.N. to NYC may simply indicate that this relationship has no strong emotional valence. These are revisited later.

Information organized in thought can be holistic and multidimensional (at least initially). However, when assembled for communication, it must be arranged in a serial way due to the sequential structure of language. Thus, the available pointers we mobilize become of particular importance in determining the likely linkages that are used in actively composing narrative. Moreover, in some cases the available pointers place constraints on how the sequence can be organized. If there are few available pointers, the options for organizing a coherent cross-linked sequence of statements may be minimal. Therefore, we see once again the importance of having multiple meaningful pointers associated with each labeled category in memory. The richer the array of pointers associated with each label, and those to which they point, the more likely the person can mobilize a creative, coherent, and thoroughly integrated communication. 


\section{Summary of the CLP Model}

In sum, the CLP model assumes that working memory, associated with the frontal lobes, is largely responsible for the voluntary recall and organization of information from long-term memory (in addition to any new communicated information). The perceived context, that occasions the recall, establishes the organizational rule or principle in working memory used to access and organize relevant information. Because there are reciprocal cross-linking nerve tracts between the frontal lobes and regions of the cortex where memories are stored, the frontal lobe not only accesses the stored information, but also can control the attentional focus. Thus, the frontal lobes have executive control over what is recalled and the degree of detail. During organization of the recalled content, information with clearly identified labels can be processed most efficiently. This is due to the limited capacity of working memory. The more information units that are "chunked" or grouped under clearly accessible representative labels, the more likely they can be processed rapidly and effectively in working memory. Labeled categories theoretically bear pointers that indicate the variety of other labeled ideational entities that can be organizationally linked with each one during construction on information networks in memory. The more varied and numerous the pointers associated with each labeled category, the more likely that the individual will be able to create a richly coherent and interconnected narrative. The fewer pointers available for each labeled category, the less potential for making richly interconnected representations of the recalled information. By definition, knowledge is the meaningful recall of information, assembled in some organized way. The larger the number of interrelationships among the units of information organized in memory, the more likely the individual will have multiple ways of accessing and applying the information in a meaningful way. Hence, the more likely he or she will be able to think about it constructively and creatively.

\section{Applications to Teaching and Learning}

A considerable amount of published evidence indicates that students who organize information with multiple interrelationships during recall are more academically successful. This includes more accurate and complete recall of learned material (e.g., Anderson, 2009a; Anderson \& Contino, 2010; Dhindsa et al., 2011; Tsai, 2003), evidence of higher level conceptualization and thinking during recall (Bischoff \& Anderson, 2001), and enhanced skill in applying knowledge in analytical tasks such as critical thinking and analysis of data (Anderson, 2009a; Bischoff, 1999; Tsai, 2003). The research methodology is based on a coding scheme that is used to analyze recorded 
oral or written narrative. The flow of the discourse is analyzed for evidence of recursive relational references to content that the student previously introduced in the stream of narrative. The analysis method is known as flow map analysis. For research purposes, recall is elicited using a carefully described protocol where the context is clearly presented (e.g., Anderson, 2009a).

\section{The CLP Theory in Practical Perspective}

According to the CLP theory, there are several major considerations that curriculum designers and teachers need to keep in mind to enhance students' ability to organize and encode information in memory in such a way as to facilitate its efficient multi-relational (networked) recall. The use of a clearly defined context to situate the initial learning is of seminal importance. Context can be established by using a variety of methods. Anchored instruction (e.g., Bransford et al., 1990; NASA, n.d.) that introduces the topic in a clearly organized, episodic (everyday) example can be very effective. A current social issues topic, a problem in environmental pollution, a recent news event that can be video-presented to the class, or an example of an applied scientific accomplishment, are examples from various disciplines that can act as the contextual situation as relevant to the topic of the lesson. It is very important that the context is sufficiently generalized to facilitate mobilization later in a variety of relevant circumstances, including topics of current events and social discourse. Once the situation is well defined as much as possible, subsequent learning activities should be organized to continuously address the context as a theme, thus sparing excessive demand on working memory. Other means include using a visual organizing display. This may include a diagram of suitable generality, but not overly detailed, that otherwise may tax working memory. Student small-group activities that involve making organizing visual maps (such as network diagrams, charts, etcetera) may establish a concrete referent that can be used as a context for organizing information in memory as the lesson unfolds. More on this at a later point.

Given the limited capacity of working memory, it is important that the contextual theme is revisited as the class experience progresses. The context initially presented is basically a guide or map of "where we are going." Subsequently, to enhance effective contextual binding of new information, it is important to help the learners understand "where we are now" by regularly relating the new information to the initial contextual organizing device. Furthermore, a clear plan is important to identify the major categories of ideas to be learned as the lesson proceeds, and each idea should be identified by an easily remembered topic word or phrase "the label." For example, in a lesson on food webs in ecology, the key ideas may include the major 
biotic components of the web. These are: primary producers (e.g., green plants), primary consumers, and secondary consumers, etcetera. These terms, properly defined, can serve as labels for the key ideas needed to develop a coherent conception of food webs. Each of these labels, moreover, should be well established with "pointers," the logical linking attributes that relate it to one or more other labeled ideas. A careful plan for a lesson requires some forethought about what are the most important and succinct linking attributes (serving as pointers) that can be carefully presented or elicited from the students as each labeled idea is introduced. Students need to engage actively in discussing the labeled idea and its linking attributes, perhaps by assembling them as the lesson unfolds into network diagrams related to the context for the lesson, or by other organizing diagrams, story lines, etcetera that permit the labeled ideas and their linkers to be effectively instantiated in memory. Again, constant reference to the context is critical as each labeled idea and linkers are introduced.

Returning to our current example from ecology, when the concept of a primary producer (green plants or other photosynthetic organisms) is fully developed, a logical progression is to explore the role of primary producers in sustaining other living things by eliciting examples of organisms that consume plants. It is important to elicit as many linkages as possible within the food chain from primary producers to other predatory organisms, thus increasing the number of possible pointers in memory. The predatory organisms can be labeled as primary consumers, those that feed on primary producers, but also including reciprocal (backward directed) pointers as well. For example, the primary consumers also control the population size of the primary producers by preying on them, thus these backward-directed links (pointers) need to be fully discussed and developed. Forward and backward, reciprocal pointers are significantly important in helping students develop strongly linked knowledge networks. They also provide plasticity in recalling information that allows for multiple relational thinking as students mobilize and organize information for recall in working memory. For example, reconsider our sequential order based on size of the Great Lakes. A very different order would be appropriate from the perspective of economic geography, if for example we were asked to explain the transport of goods and materials by ship from the Canadian Maritimes to the midlands; i.e., Lake Ontario through Lake Erie and thence into Lake Huron with further transport into Lake Superior and ultimately into Lake Michigan. For a coherent and interrelated exposition of information based on both the physical and economic geography of the Great Lakes, a complete set of reciprocal (bidirectional) pointers in memory would be required. While working through the complete sequence of a lesson such as the food web, or one on the Great Lakes, a diagram such as the one illustrated in Figure 2 or 3, can be constructed, either using digital projection applications (e.g., PowerPoint) or of course white boards, or other non-digital media. 


\section{Some Recommendations to Remember}

The important points to remember are to: (1) present the context continuously as the lesson unfolds, (2) clearly establish the relationship of each labeled idea to the broader context in addition to exploring with the students the particular linking pointers that are associated with each of the labeled ideas, and ( 3 ) use some form of visual representation to exhibit the network of ideas as they are unfolding. Multimodal representations, including where possible assembling images or symbols of the linked ideas that the students manipulate and arrange on charts or maps (individually or in small groups), are an important consideration. We know the richer the mode of representation (verbal, visual, psychomotor/manipulative) that the students encode in memory as the lesson unfolds, the more likely that they will subsequently have greater probability of accessing information for recall. The issue of reciprocal relations is of some considerable importance, especially for topics where there is potentially a hierarchical, linear sequential, or other salient ordering pattern. Some students tend to encode the information in a strictly unidirectional way. For example, in hierarchical systems such as social, political, or cultural, some students tend to link information only from the most super-ordinate or antecedent categories toward the more subsumed or subsequent occurring components. This strictly forward linking of ideas limits accessibility in networking of information where multiple and diverse linking of ideas requires multi-directional logical organization. For example, when we analyze the logical flow of ideas in students' narrative using the flow-map technique, students with less networking of information tend to have fewer instances of recursive relational utterances as the narrative unfolds. Because they apparently lack sufficient knowledge of reciprocal relations among ideas, they have difficulty in crossrelating their thoughts as their narrative unfolds. Acquisition of accurate pointers is essential to subsequent correct recall. We all have probably encountered students who for some reason learn information with incorrect linkages, and upon recall embark on an explanation that is clearly "off track" relative to accurate knowledge in the field.

\section{Some Approaches to Applying the CPL Model}

A range of devices (such as various mind-mapping techniques) can be used to enhance students' networking of ideas (e.g., Dhindsa et al., 2011; Mind Tools, 2001). We have found, however, that a particular approach of this kind, called web diagrams, can be effective in improving knowledge networking, especially for students who are initially less adept (Anderson \& Contino, 2010). Fundamentally, this is a diagram (e.g., Figure 3) constructed by students. 




Fig. 3: Web diagram skeletal framework ${ }^{3}$

It includes iconic visual elements and written verbal linking connections among the labeled visual elements. An example of such a diagram for basic government organization and functions is presented as Figure 3. Students are supplied with a relatively large sheet of paper (e.g., $11 \times 17$ inches) and a set of small paste-on images representing the major conceptual ideas to be interrelated (here some major branches of government). The iconic images are placed on the paper near the periphery in a pattern that permits inserting written linkages, completed thoughts (clauses or sentences), along the connecting arrows. In contrast to some mind-mapping techniques that use single words such as predicates to link the nodes, we encourage students to use completed thoughts, especially including language that expresses bidirectional relations. For example, in Figure 3, the Executive Office link (A) to the legislative branch of government may include statements such as "Executive decision-making, but subject to laws made by the legislators." Similarly, link (B) from the Executive Office to the Judiciary, may include "Separation of powers ensures the Executive office is subjected to the decision of the courts." Or, for the link (C) from the Executive to the Dept. of State, the written statement may be "Executive sets policy within law and in turn is informed of national status." If digital media are available, the students can be given the iconic tabs as small digital images to be pasted into a word-processing or image-processing application to create their web diagrams in digital format. When the web diagram is completed, the student(s) who prepared it individually, or as a small group, should be encouraged to write an essay or make an oral presentation that explicates the multirelations shown in their web diagram. We have found that students who complete such coherent diagrams, and use them as guides in writing narrative, exhibit higher quality of thinking, more coherent discourse, and increased generality and conceptual level of thought. Thus, in addition to improving their integrated knowledge of a subject, they also may improve their literacy. We have used this successfully with adolescent students, and it very likely can be adapted for younger students. 


\section{Keeping Context and Affective Valence Clearly in Mind}

Given the important role of the context for initial encoding and also for subsequent organized recall of information, it is important to establish the generalized properties of the context. That is, to help students through discussion or other deep intellectual involvement, to understand what features of the contextual situation especially characterize it, and how other examples of the particular context can be recognized on future occasions. For instance, in a civics class, if a court case is the particular context used to establish the network of ideas, it is important to include at least a final summarizing discussion of what the salient features of the case are. In addition, help students understand what range of other legal situations or social circumstances would also be considered as belonging to this class of phenomena. Unless this refining step is made, students may not be able to mobilize and apply their integrated network knowledge on subsequent occasions where the eliciting cues are from different, but relevant, contextual situations. Broadening the identification of the context, therefore, is a very important consolidating and generalizing step in the learning process.

As promised, we return to a discussion about the emotional valence associated with the pointers. It is important to emphasize the merits of including appropriate opportunities to address affective dimensions in teaching. For example, in the social sciences and humanities, excerpts of poetry, literature, and musical genre of interest to the students can be included to lend additional affective strength to the topics to be encoded in memory. Certainly, moral issues should be considered with adequate opportunity to express rational emotional responses to situations that affect individual or collective well-being in society. This is equally true in sciences, as well as the humanities, where issues of science, technology, and society provide opportunity for moments of reflective critical analysis of the role of science in society, including moral and ethical dimensions of proper and improper uses of science and its applications.

\section{Summary}

Modern neuroscience has partially confirmed, and more fully amplified, psychological explanations of how we learn and respond to our environments, especially the role of cognition in perceiving and internally representing experiences. We now know that the frontal lobes are the site of major information processing in working memory, guiding the recall of information from long-term memory to be 
merged with current incoming information from the environment to dynamically create organized representations of experience. Nerve fiber projections interconnecting the frontal lobes with sensory portions of the brain permit controlled recall of stored information, regulate the focus of attention, and using contextual organizing rules or principles organize the information into a form to be communicated. The kinds of conceptual linkages made, and networking richness, depend on the number and diversity of associated pointers that have been associated with each major idea. The pointers are used to direct the composite linkages among the ideas constructed in working memory. The situational context determines what organizing rules are applied and what pointers are likely to be mobilized in constructing a coherent representation of knowledge to be communicated. Pointers also carry emotional valence, and this can be a useful adjunct to the cognitive meanings associated with the categories and their pointers during learning. Working memory is limited in capacity, so it is important during learning to keep a consistent context in perspective, make clear the kinds and variations of pointers that can be associated with each idea to be learned, and help students to use these cognitive tools to better organize and store information in memory for later coherent recall. Multimodal teaching and learning can facilitate the richness of the context and the variety of pointers in memory. Modern strategies of active, hands-on learning, and teaching (e.g., mind maps and web diagrams) that facilitate networking of ideas during learning can be used to enhance students' preparation to encode memorized meaningful information in a more stable way. Thus, it can be recalled, better organized, and applied more effectively at a later time.

\section{Notes}

1. Frontal lobe is a site of working memory and executive control functions. Temporal lobes (left and right) mediate verbal learning and formation of conceptual information, including a region for auditory perception. The occipital lobe is the site for visual perception. Somatosensory cortex receives tactile and body sensory information. The motor cortex, immediately adjacent to the frontal lobes, controls movement. The limbic system mediating formation of long-term memories, and at other sites emotion, lies deep within the brain at the level of the temporal lobes. The bidirectional arrows indicate nerve fibers that link the frontal lobes to the sensory areas of the brain where information is stored as described in the text. 
2. A diagram illustrating interconnected information in networks as represented by the CLP model, with labeled information entities (e.g., Washington, D.C.) and others interconnected by arrows representing pointers that are mobilized in memory to mediate making linkages during construction of information networks in working memory. There are two contextual fields, enclosed by dashed lines that occasion the knowledge recall: socio-political (right panel) and geographic (left panel). See text for details.

3. An example of a web diagram skeletal framework for knowledge about basic government organization and functions. The labeled pictorial (iconic) entities and the interconnecting arrows (pointers) are applied as a student might prepare them for the next step of inscribing written clauses or sentences along each arrow describing each of the interrelationships. See text for examples of possible inscribed sentences for the links $A, B$, and $C$.

\section{References}

Anderson, O. R. (2009a). The role of knowledge network structures in learning scientific habits of mind: Higher order thinking and inquiry skills. In I. M. Saleh \& M. S. Khine (Eds.), Fostering Scientific Habits of Mind: Pedagogical Knowledge and Best Practices in Science Education (pp. 59-82). Rotterdam, The Netherlands: Sense Publishers.

Anderson, O. R. (2009b). Neurocognitive theory and constructivism in science education: A review of neurobiological, cognitive and cultural perspectives. Brunei International Journal of Mathematics and Science Education, 1, 1-32.

Anderson, O. R., \& Contino, J. (2010). A study of teacher-mediated enhancement of students' organization of Earth science knowledge using web diagrams as a teaching device. Journal of Research in Science Teacher Education, 21, 683-701.

Bischoff, P. J. (1999). The development of ideational networks, systemic knowledge, and application abilities in preservice elementary teachers who studied a laboratory unit on ecology. Georgia Journal of Science, 57, 210-223.
Bischoff, P. J., \& Anderson, O. R. (2001). Development of knowledge frameworks and higher order cognitive operations among secondary school students who studied a unit on ecology. Journal of Biological Education, 35, 81-88.

Bransford, J. D., \& Cognition and Technology Group at Vanderbilt (CTGV). (1990). Anchored instruction: Why we need it and how technology can help. In D. Nix \& R. Spiro (Eds.), Cognition, education and multimedia. Hillsdale, NJ: Erlbaum Associates.

Dhindsa, H. S., Kasim, M., \& Anderson, O. R. (2011). Constructivist-visual mind map teaching approach and the quality of students' cognitive structures. Journal of Science Education and Technology, 20, 186-200.

Gathercole, S. E., \& Alloway T. P. (2008). Working memory and learning: A practical guide. London: Sage Publications Ltd.

Holistic-educator (n.d.). Findings from brain research on 'neuroscience': Implications for learning theory. Retrieved May 16, 2011, from http://www.holisticeducator.com/ neuroscience.htm 
Jonides, J., Lewis, R. L., Nee, D. E., Lustig, C. A., Berman M. G., \& Moore K. S. (2008). The mind and brain of short-term memory. Annual Review of Psychology, 59, 193-224.

Mind Tools (2001). Mind maps: A powerful approach to note-taking. Retrieved May 15, 2011, from http://www.mindtools.com/ pages/article/newISS_01.htm

NASA (n.d.). Anchored instruction. Retrieved May 15, 2011, from http://www.ed.psu. edu/NASA/achrtxt.html
Thorne, G. (2006). 10 strategies to enhance students' memory. Retrieved May 15, 2011, from http://www.cdl.org/resource-library /articles/memory_strategies_May06.php

Tsai, C.-C. (2003). Using a "conflict map" as an instructional tool to change student alternative conceptions in simple series electric-circuits. International Journal of Science Education, 25, 307-327.

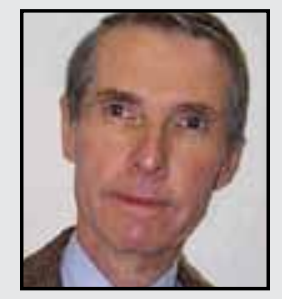

O. Roger Anderson is Chair of the Department of Mathematics, Science and Technology at Teachers College and a Senior Research Scientist at Columbia University, New York. His research in education has particularly focused on cognitive and neuroscientific perspectives on teaching and learning, especially in science education. Here, he presents a more general view of neurocognitive theory as applied to knowledge organization for effective recall and application appropriate to a broader range of subject matter disciplines.

LINKTO:

http://www.tc.columbia.edu/academics/?facid=ora1 